\title{
Progressive Fetal Distress Estimation by Characterization of Fetal Heart Rate Decelerations Response Based on Signal Variability in Cardiotocographic Recordings
}

\author{
Patricio Fuentealba, Alfredo Illanes, Frank Ortmeier \\ Otto-von-Guericke University, Magdeburg, Germany
}

\begin{abstract}
In the current clinical practice, Cardiotocograph (CTG) is a standard and widely used tool for fetal surveillance. However, CTG interpretation is difficult since it involves human diagnosis of different patterns in highly complex signals. Fetal heart rate (FHR) decelerations and variability are known to be the most significant and difficult patterns to assess by the clinical staff. The main goal of this work is to analyze the fetal distress by tracking the evolution of the dynamical changes occurring in the CTG recording. The idea is to consider the direct input/output relationship between uterine contraction $(U C)$ and FHR signals by the characterization of FHR decelerations in terms of their signal variability as a sign of the fetal response corresponding to a UC event. Results show that the progression of the decelerations response over time can help the observer to track fetal distress.
\end{abstract}

\section{Introduction}

During labor, fetal monitoring is essential to help clinicians for timely intervention in order to prevent potential adverse outcomes resulting from decreased oxygen supply [1]. Fetal monitoring is usually based on the joint recording of FHR and UC signals acquired by CTG, which is the most widely used tool for fetal welfare assessment during the perinatal period. In clinical practice, the CTG interpretation relies mainly on four FHR signal patterns: baseline, accelerations, decelerations and variability. However, the analysis of these patterns is difficult because it involves a human interpretation of highly complex signals leading to a wide intra- and inter-observer disagreement [1].

In order to increase the objectivity and improve the CTG interpretation, several medical guidelines [2] and expert systems (ES) [3] have been proposed. Nevertheless, guidelines still lack consensus in many key aspects due mainly to their complexity and ES have not shown evidence that they improve fetal welfare assessment compared with the classic CTG analysis [3]. According to the literature [4], the observer disagreement is related mainly with the interpretation of FHR decelerations and variability. For that, many works have been proposed to extract hidden features in the FHR signal using signal processing techniques such as FFT, Short Time Fourier Transform, AR modeling and Continuous and Discrete Wavelets Transform [5]. Most of them do not take into account the input/output relationship between UC and FHR signals. In this context, some works have been proposed in order to consider CTG as an input/output system [6,7], however, they do not consider the progression of decelerations along the FHR signal.

In this work, we propose a new methodology of CTG analysis based on the evolution of the fetal response by the characterization of FHR decelerations over time. The main idea is to analyze the direct input/output relationship between UC and FHR signals in order to track the progression of the fetal condition based on the variability during deceleration episodes as a response to a UC event. For this purpose, deceleration episodes are first identified according to their location, length and depth making use of a progressive baseline. Then, in each identified episode the variability is estimated as a sign of the fetal response corresponding to a UC event that trigger such episode.

Results show that both the progressive baseline and decelerations can describe different time-variant dynamics and their evolution over time can help the observer to track fetal distress behavior during labor.

\section{Method}

The analysis is performed using CTG recordings extracted from the CTU-UHB Intrapartum Cardiotocography Database freely available on the PhysioNet Website [8] and the principles of the approach is explained making use of the FHR and UC signals shown in Figure 1.

\subsection{Fetal heart rate signal pre-processing}

The acquisition of CTG signals involves different types of artifacts such as loss of data and outliers. For that, following [9], values less than $50 \mathrm{bpm}$ and more than 200 

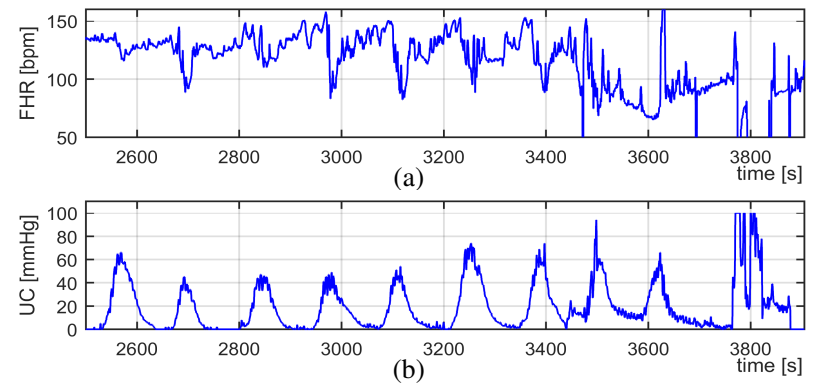

Figure 1: CTG recording nb. 1022m (Last $1400 s$ ), $\mathrm{pH}=7.28$. (a) Raw FHR signal; (b) Raw UC signal.

bpm are removed from the FHR signal and then the segments are interpolated using a Hermite spline method.

The FHR decelerations lie in the frequency range between 0 and $0.05 \mathrm{~Hz}$ involving non-linear characteristics [5]. For that, a non-linear median filter [10] is applied over a sliding window of 10 seconds length (see Figure 2a).

\subsection{Identification of decelerations}

The FHR decelerations are identified making use of a progressive baseline and considering UCs as stimuli that trigger deceleration episodes as explained in the following.

The first step for decelerations identification is performed following the method proposed in [11], which allows the detection of deceleration episodes through the use of a floating-line. The detected episodes of more than $15 \mathrm{bpm}$ in amplitude and more than 15 seconds length [2] (evident decelerations) are then parameterized according to their location, length and depth. Figure $2 b$ shows the floating-line and the evident deceleration episodes.

The second step allows the identification of episodes that were not recognized as evident decelerations but that they are certainly a response to a UC event. Those episodes (called $U C$-segments) are identified following the criteria proposed in [7] where the UC-segments start 7 seconds before the UC apex and end 50 seconds after it. For that, first, $\mathrm{UC}$ apexes of pronounced amplitude $(\geq 30 \mathrm{~mm} \mathrm{Hg}$ ) are detected (see Figure 2c) and then, when an evident deceleration episode has not been identified but a UC event is detected, its corresponding UC-segment is also considered for the analysis (see Figure 2d).

\subsection{Variability of decelerations}

After deceleration episodes have been identified, they are described by their FHR variability, which is represented by the difference between the interpolated and the filtered FHR signal described in Section 2.1. Therefore, in each deceleration episode the variability is calculated as the average of the peak-to-peak amplitude of the interpo-
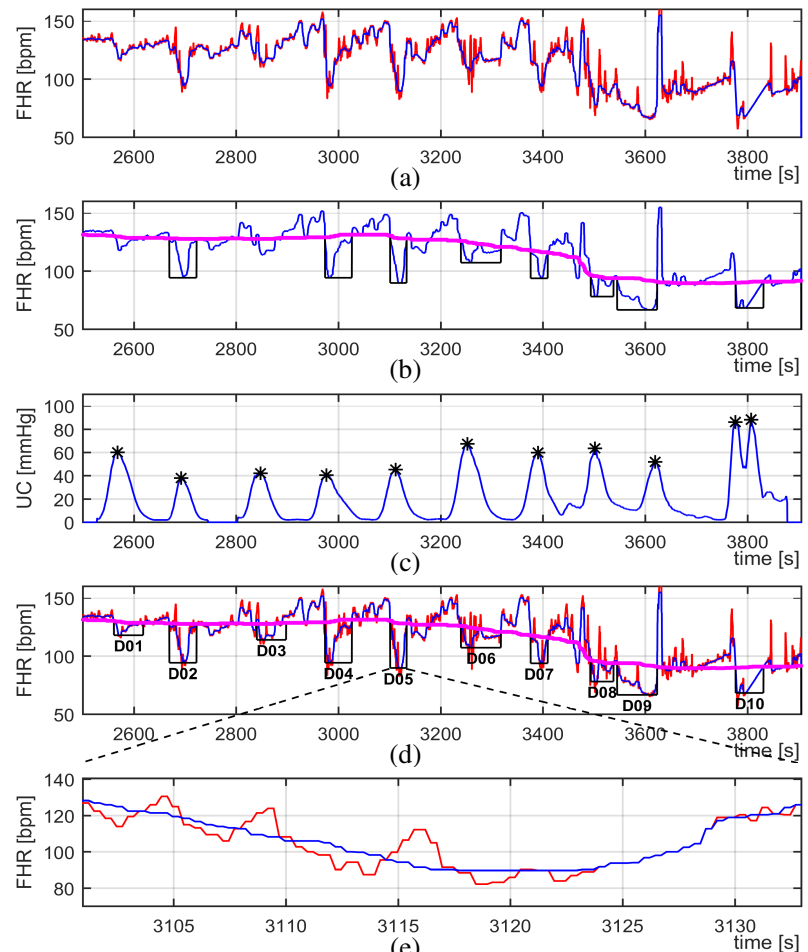

(e)
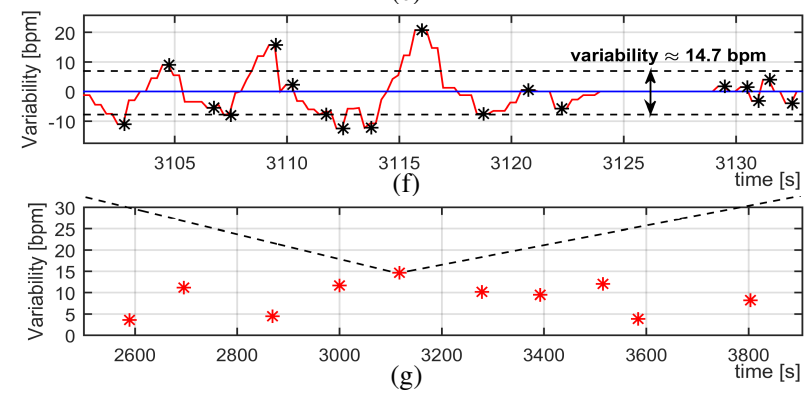

Figure 2: Proposed method. (a) Interpolated (red) and filtered FHR signal (blue); (b) Floating-line (magenta) and evident deceleration episodes (black); (c) Filtered UC signal (blue) and the intensity of its UC events (black dots); (d) 10 identified deceleration episodes (black); (e) Zoom of deceleration D05; (f) FHR variability estimation of D05; (g) FHR variability calculated in 10 deceleration episodes.

lated FHR signal, by taking as reference level the filtered FHR signal. Figures $2 \mathrm{e}$ and $2 \mathrm{f}$ illustrate a graphic representation of this operation, where a zoom of a particular deceleration episode is shown.

\section{Results}

The analysis is focused on the evolution of the floating-line and the FHR variability during decelerations. The main idea is to analyze the progression of these patterns as a sign of the fetal response corresponding to a UC in order to estimate the fetal distress. 

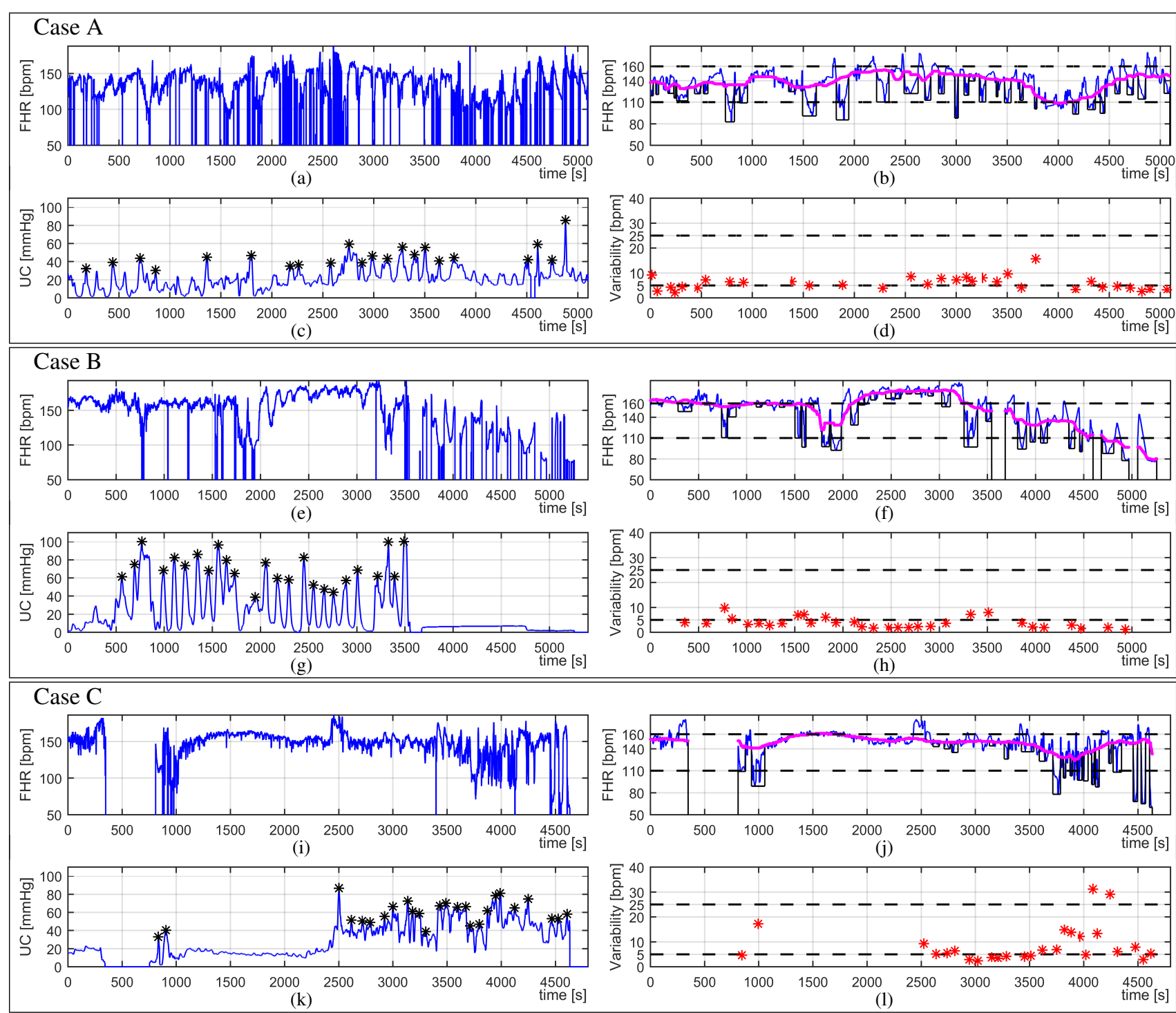

Figure 3: Three representative cases of CTG. Case A: CTG recording nb. $1490 \mathrm{~m}, \mathrm{pH}=6.93$, pathological. Case B: CTG recording nb. $1017 \mathrm{~m}, \mathrm{pH}=7.00$, pathological. Case $\mathrm{C}$ : CTG recording nb. $1332 \mathrm{~m}, \mathrm{pH}=7.27$, normal. (a)(e)(i) Raw FHR signal; (b)(f)(j) Filtered FHR signal (blue), floating-line (magenta) and reference levels of bradycardia and tachycardia (dotted lines); (c)(g)(k) Filtered UC signal (blue) and the intensity of its UC events (black dots); (d)(h)(l) FHR variability at each deceleration and reference levels of reduced and increased variability (dotted lines) [2].

Figure 3 shows three representative cases selected according to their $\mathrm{pH}$ value, which is considered as a standard measurement for fetal acidemia. Values of $\mathrm{pH}$ less or equal than 7.05 indicate a pathological fetus and values greater or equal than 7.25 indicate a normal fetal condition [8]. Cases A and B correspond to pathological fetuses and case $\mathrm{C}$ represents a normal fetal condition.

Results show that both the floating-line and variability during decelerations exhibit different time-variant dynamics for the three cases. Case A shows an interesting exam- ple, where a repetitive set of UCs triggers consecutive deceleration episodes. Figure $3 \mathrm{~b}$ shows that the floating-line presents an unstable behavior along the signal involving a bradycardia episode (below $110 \mathrm{bpm}$ ) around $4000 \mathrm{sec}-$ onds. In Figure 3d, we can observe that decelerations show different responses over time described by values of reduced (values below than $5 \mathrm{bpm}$ ) and normal variability. Particularly, during the last 1000 seconds decelerations exhibit a sustained reduced variability, which together with the bradycardia episode could indicate a high fetal distress. 
Concerning to the case 2, in Figure $3 f$ we can observe that the floating-line presents an unstable behavior involving a long episode of tachycardia (values above $160 \mathrm{bpm}$ ) between 2200 and 3300 seconds. After this episode, it exhibits a decreasing slope and from 4700 seconds it involves a bradycardia episode. Figure $3 \mathrm{~h}$ shows that the variability involves mainly values oscillating close to $5 \mathrm{bpm}$ and in the last 1500 seconds it shows a sustained reduced variability. This behavior in addition with the floating-line response could indicate a high fetal distress.

Results displayed in case $\mathrm{C}$ strongly differ from the ones presented previously, where both the floating-line and variability exhibit a completely different behavior. In this third case, the floating-line shows a more stable trend whose values are inside the normal range (between 110 and 160 bpm). Additionally, the variability during decelerations shows mainly values considered normal (between 5 and $25 \mathrm{bpm}$ ). In particular, in the last 1200 seconds, most of deceleration episodes show a normal variability in contrast to the opposite behavior of the previous cases. The information described about the variability together with the normal floating-line behavior could indicate a normal fetal condition.

It is important to note that the analysis described above coincides with the $\mathrm{pH}$ outcome assessment because the first two examples are labeled with $\mathrm{pH}$ values of 6.93 and 7.00 , respectively (pathological) and the last example is labeled with a $\mathrm{pH}$ value of 7.27 (normal).

\section{Conclusion}

In this work, we proposed the analysis of the CTG recording based on the characterization of FHR decelerations described by their variability as a sign of the fetal response corresponding to a UC event. The main idea was to study the behavior of these CTG patterns over time considering their evolution in order to predict the fetal distress.

Results illustrated that both the floating-line and variability during decelerations can describe different time-variant dynamics and their progression over time can help the observer to track the fetal distress during labor.

Considering that FHR deceleration episodes and variability are known to be the most significant and difficult CTG patterns to assess by the clinical staff, these results open perspectives for classification of non-reassuring CTG recordings from a progressive analysis point of view.

As a further step, we propose to classify CTG signals by the characterization of the variability during decelerations based on frequency analysis using digital signal processing techniques. The main idea would be to extract signal features from deceleration episodes described by their variability and making use of the floating-line to track the fetal condition in order to obtain an indicator of fetal distress.

\section{Acknowledgements}

Research funded by the National Commission for Scientific and Technological Research CONICYT, Chilean National Scholarship Program for Graduate Studies.

\section{References}

[1] Ayres-de Campos D, Bernardes J. Twenty-five years after the figo guidelines for the use of fetal monitoring: Time for a simplified approach? International Journal of Gynecology Obstetrics 2010;110(1):1-6.

[2] Ayres-de Campos D, Spong CY, Chandraharan E, et al. Figo consensus guidelines on intrapartum fetal monitoring: Cardiotocography. International Journal of Gynecology Obstetrics 2015;131(1):13-24.

[3] Nunes I, Ayres-de Campos D. Computer analysis of foetal monitoring signals. Best Practice Research Clinical Obstetrics Gynaecology 2016;30:68-78.

[4] Hruban L, Spilka J, Chudáček, et al. Agreement on intrapartum cardiotocogram recordings between expert obstetricians. Journal of evaluation in clinical practice 2015;

[5] Haritopoulos M, Illanes A, Nandi AK. Survey on cardiotocography feature extraction algorithms for foetal welfare assessment. In XIV Mediterranean Conference on Medical and Biological Engineering and Computing 2016. Springer, 2016; 1193-1198.

[6] Warrick PA, Hamilton EF, Precup D, Kearney RE. Classification of normal and hypoxic fetuses from systems modeling of intrapartum cardiotocography. IEEE Transactions on Biomedical Engineering 2010;57(4):771-779.

[7] Romano M, Bifulco P, Cesarelli M, Sansone M, Bracale M. Foetal heart rate power spectrum response to uterine contraction. Medical and Biological Engineering and Computing 2006;44(3):188-201.

[8] Chudáček V, Spilka J, Burša M, Janku P, Hruban L, Huptych M, Lhotská L. Open access intrapartum CTG database. BMC pregnancy and childbirth 2014;14(1):16.

[9] Spilka J, Georgoulas G, Karvelis P, Oikonomou VP, Chudáček V, Stylios C, Lhotská L, Janku P. Automatic evaluation of FHR recordings from CTU-UHB CTG database. In Information Technology in Bio-and Medical Informatics. Springer, 2013; 47-61.

[10] Sameni R, Shamsollahi M, Jutten C. Model-based bayesian filtering of cardiac contaminants from biomedical recordings. Physiological Measurement 2008;29(5):595.

[11] Fuentealba P, Illanes A, Ortmeier F. Analysis of the foetal heart rate in cardiotocographic recordings through a progressive characterization of decelerations. Current Directions in Biomedical Engineering 2017;3(2):423-427.

Address for correspondence:

Patricio Fuentealba

Faculty of Computer Science

Universitätsplatz 2, 39106, Magdeburg, Germany

patricio.fuentealba@ovgu.de 